International Journal on Bioinformatics \& Biosciences (IJBB) Vol.2, No.4, December 2012

\title{
Perspectives on Computer Assisted Surgery (CAS) IN MINIMALLY INVASIVE SURGERY AND THE ROLE OF THE CAS SYSTEM NURSE
}

\author{
João Fradinho Oliveira ${ }^{1}$ Hugo Capote ${ }^{2}$ José Blas Pagador $^{3}$ José Luis Moyano $^{4}$ \\ Cuevas $^{3}$ Francisco Miguel Sánchez Margallo ${ }^{4}$ \\ ${ }^{1} \mathrm{C} 3 \mathrm{i} /$ Instituto Politécnico de Portalegre, Portalegre, Portugal \\ jfoliveiralestgp.pt \\ ${ }^{2}$ Hospital Dr. José Maria Grande in Portalegre, Portugal \\ hugocapote@gmail.com \\ ${ }^{3}$ Bioengineering and Health Technology Unit, Jesus Uson Minimally Invasive Surgery \\ Center, Caceres, Spain \\ \{jbpagador, jmoyano\}@ccmijesususon.com \\ ${ }^{4}$ Jesus Uson Minimally Invasive Surgery Center, Caceres, Spain \\ msanchez@ccmijesususon.com
}

\begin{abstract}
Minimally invasive surgery has become a widely used surgical technique that improves patients' safety and optimizes health resources. Specifically, laparoscopic surgery uses small incisions through the abdomen to operate, for example a liver, with minimal postoperative complications and faster recoveries. However, the cognitive load and surgical skills required by surgeons increase when compared to open surgery. For these reasons, specific training models and new technologies are needed to assist surgeons. One of this kind of systems are Computer Assisted Surgery systems that enable the display of computer generated imagery of pre- and intra-operative data to assist surgeons during its surgical procedures, but in many hospitals either they are not used at a basic level due to socio-economic challenges or components still face considerable technical challenges that must be solved. In this paper we list some of the difficulties that CAS components face during a laparoscopic surgery and highlight the benefits that a new job profile, the computer assisted surgery system nurse could bring in several modelling and tracking scenarios.
\end{abstract}

\section{KEYWORDS}

Laparoscopic surgery, 3D reconstruction, navigation, tracking, computer assisted surgery system nurse

\section{INTRODUCTION}

Laparoscopic surgery has become a gold standard technique in many surgical interventions for the last years. Mainly due to its multiple safety advantages for patients and important health cost reductions [1]. However some minimally invasive procedures are complex and need additional technologies that assist and enhance surgeon performance. In this case, Image-Guided Interventions (IGI) or Computer Assisted Surgery (CAS) are very active research fields. Haigron et. al [2] makes an overview to the newest Image Guided Therapies (IGT). In order to best understand the physical and environmental constraints present in laparoscopic surgery, we refer the reader to Figure 1 and 2. Figure 1 shows the set-up of a laparoscopic surgery with a video display and the placement of 4 trocars: endoscope (video camera unit with light) and 3 laparoscopic instruments (forceps, retractor, scissors or dissectors). 
International Journal on Bioinformatics \& Biosciences (IJBB) Vol.2, No.4, December 2012

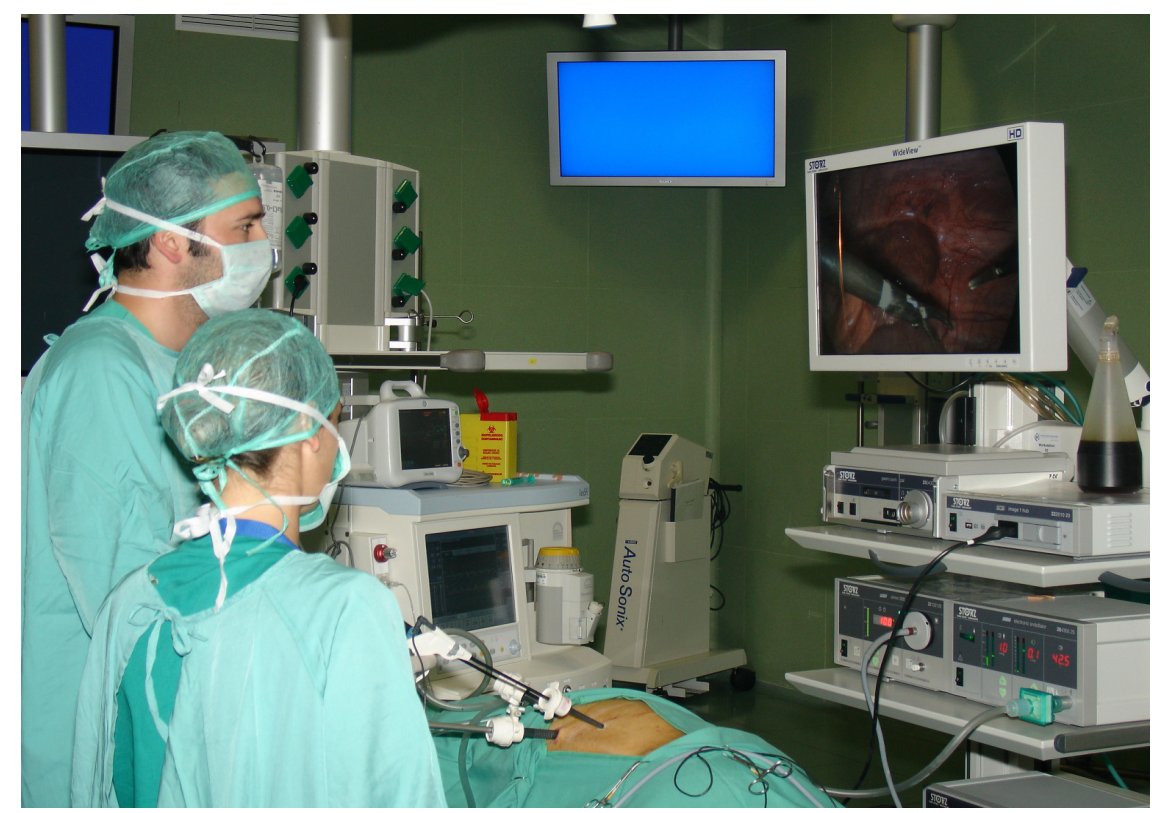

Figure 1. An example laparoscopic surgery set-up that uses 4 inserted trocars: 1) camera providing video images from inside the patient on the screen 2) scissors 3) retractor instrument to achieve adequate exposure of the surgical field 4) iron to stop bleeds

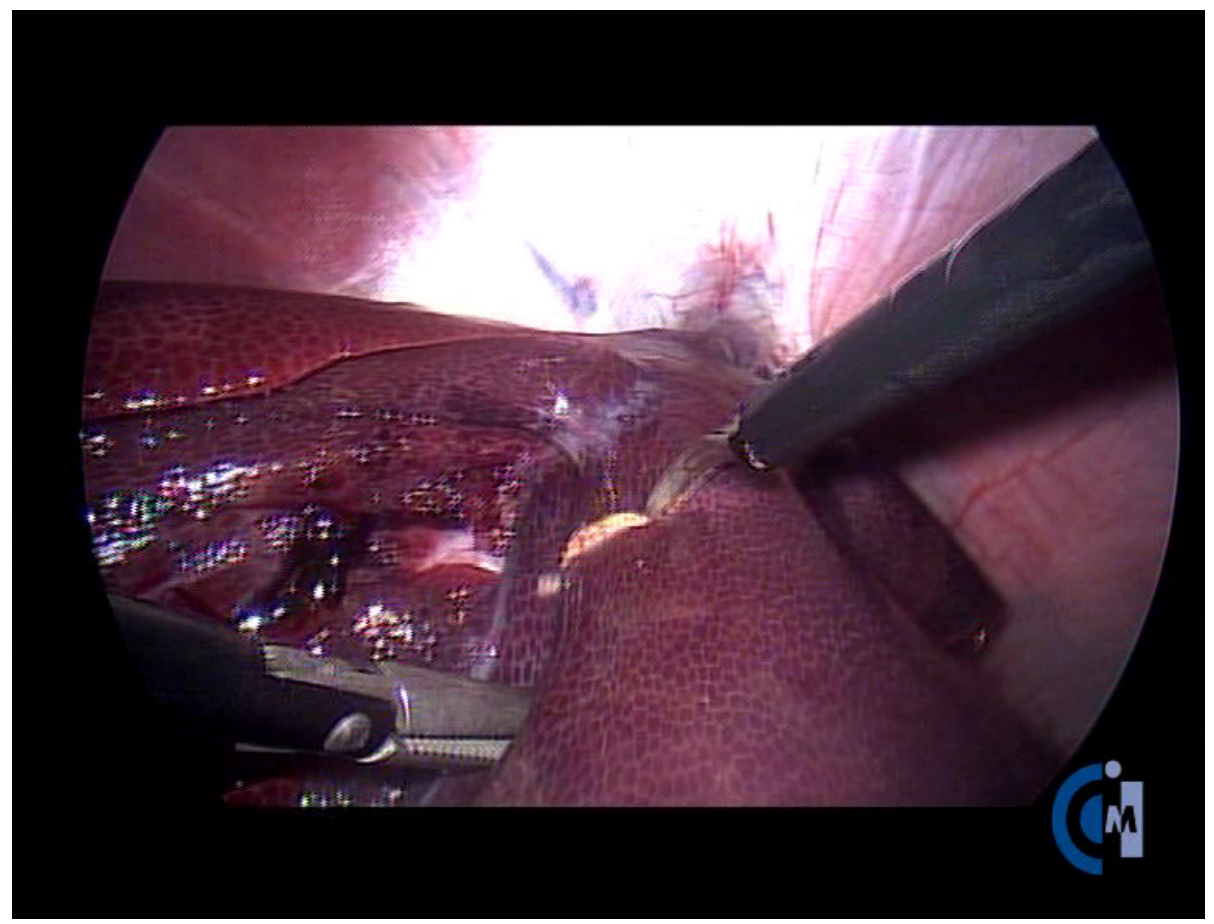

Figure 2. Typical video image of a laparoscopic surgery

In addition sometimes aspiration/flushing or haemostasis instruments are used during surgery. Currently, there are different surgical procedures that modify the number and location of trocars depending on their needs. 
Figure 2 shows an example of the surgical scene, where one can see that the field of view of the endoscopic camera is very limited, and that most of the time only partial views of the organs will be possible. Among other limitations the reduced workspace environment, cognitive load, limited tactile perception and difficulties with hand-eye coordination are the most important problems for surgeons.

The endoscopic camera can fog up or be smudged with blood during surgical procedure and has to be removed, cleaned and re-inserted. Furthermore, the focus of the camera may also require manual adjustment. In the other hand, the task of reaching the target location to be operated can be regarded as exploratory, as organs are shifting with the breathing cycle of the patient, organs need to be temporarily moved aside to access other structures and the expected anatomy can have considerable differences in shape, position, texture and colour to the one observed. Once the target area of surgery is reached, a trained surgeon can remove, clean, re-insert the camera and find the previous target with a great speed by memorizing the tactile information and actual organ placement.

Finally, in a typical operation usually a second surgeon is responsible for handling the camera, retracting the organs and assists the surgery, whilst an assisting nurse has the role of instrumenting the surgery and provides anything that is needed during the operation.

The remainder of the paper is organized as follows: in section II we broadly categorize four CAS research areas, describe some of the challenges and limitations that they face during a real operation and list four specific problems that we believe can be addressed with a new job profile, the CAS system nurse which is presented in section III. We present future work in section IV followed by conclusions.

\section{BACKGROUND \& RELATED WORK}

The most basic computer assisted surgery system shows pre-operative data (CT or US/ Figure 3 ) during the surgical procedure together with live video from the endoscopic camera. These images can include resection maps, highlights of vein structures and are useful to lessen the cognitive load of the surgeon. More sophisticated CAS systems enable the display/overlay of 3D reconstructed computer models of organs; to track the position of instruments and render them in the reference frame of pre or intra-operative images; and to program robot arms to perform precision critical tasks. Many of these complex systems use techniques from medical image processing, computer graphics, robotics, virtual and augmented reality that are adapted with varying degrees of success to the type operation at hand, set-up and image modality being used. In this section we present some of the main problems that the classical techniques in these fields face in the laparoscopic surgery context, and list four that we believe can be addressed by the CAS system nurse. 


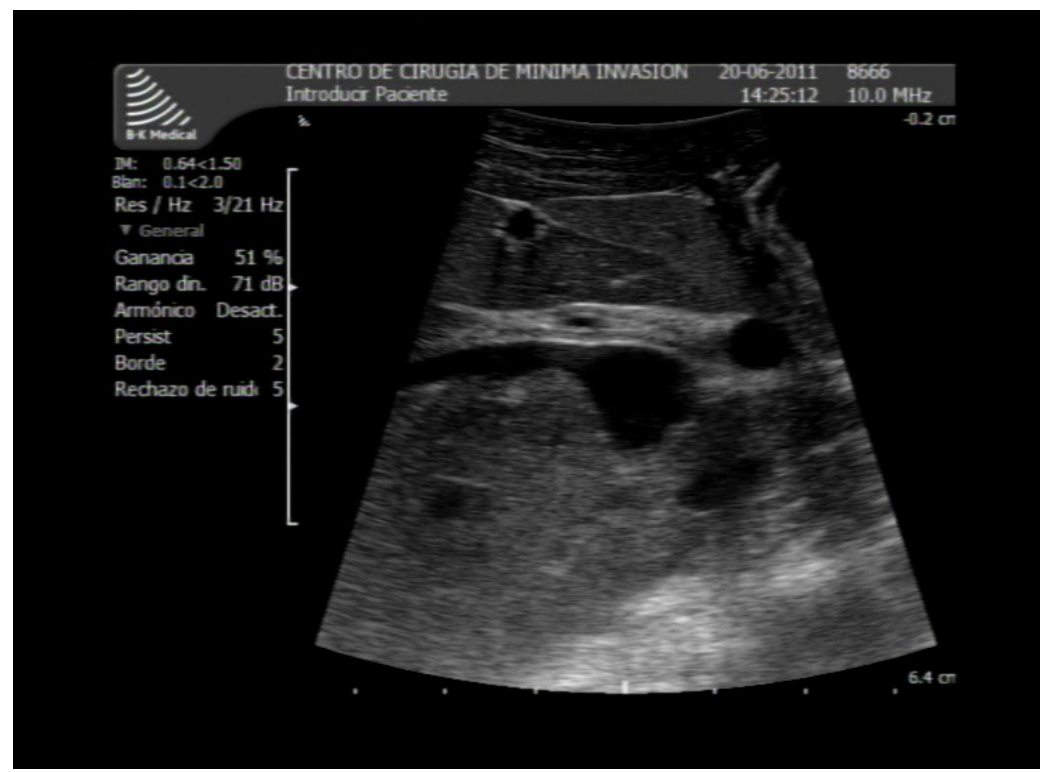

Figure 3. Ultrasound image.

\subsection{D model representation}

Computed tomography (CT) and Magnetic Resonance imaging (MRI) scanners create collections of 2D images (pixels) that can be viewed in various planes (eg. axial, coronal, sagittal) or 3D data (voxels) that can be visualized using volume rendering techniques. Whilst these representations are useful for visual inspection and calculations, iso-surface extraction methods that create polygonal surface meshes (polygons) with algorithms such as the marching cubes allow one to create a variety of other applications, for example: 3D printing of implants using standard 3D editing tools for mirroring and cutting the model, the creation of soft tissue models: (tetrahedral) using surface offsets for finite element simulation of surgical procedures with haptic devices, or used as input for Spring-Damper-Mass systems where point masses (nodes) are inter-connected by elastic springs corresponding to the edges of the initial polygonal mesh. Finally polygonal surfaces can be used for pre-operative resection map renderings.

Whilst the above representations are useful in the context of some simulations, in the context intra-operative imaging, they cannot robustly capture the $i$ ) drastic morphological changes made to soft tissues during the operation. For example, significant layers of fat or soft tissue are often cut and removed in order to access the main blood supply of the spleen during a spleen removal procedure, or when removing a tumour from a kidney the kidney can be reduced to $30 \%$ of its original volume making the original 3D model somewhat useless if ii) superimposed over corresponding parts in the live video. Furthermore real organs can bend beyond the integrity of soft models.

Implicit surfaces such as Metaballs are a somewhat more organic representation, but unfortunately there is no direct conversion from polygon model to metaballs [3], and they are not very accurate [4].

\subsection{Tracking and Registration}

Non-rigid registration relies on creating correspondences between the features in the video stream and the 3D models. Unfortunately as can be seen in Figure 2 many times only iii) partial views of organs are seen, making non-registration inherently a difficult task to be done with few 
visible markers. According to Peters and Cleary [5], one of the most important unsolved problems of IGI is non-rigid registration.

From our point of view, there are two different problems to solve in this field of research. On one side, recent research of surgical instrument tracking is focused on solving their position and velocity using video analysis techniques [6]. On the other hand, several problems with organ shift and tissue deformation are the most important challenges for the registration techniques [7]. As Baumhauer et al. [7] suggest, maybe current limitations in navigation systems could be solved using a closer collaboration between different surgical actors.

In conclusion, tracking techniques can be divided in two groups according to their use natural or artificial landmarks to track the objects of interest.

\subsection{Mental map \& Navigation}

Surgical navigation belongs to the broad field of computer assisted surgery. The intraoperative surgical navigation systems allow the surgeon to identify the anatomical structures involved and to know the spatial relationship between the target and risk structures of the surgery. However, intraoperative navigation in soft tissue presents major complications associated with the deformation of the organs during surgery. This problem leads to the situation that anatomical structures have changed from the planning done by the surgeon, so the system must adapt the preoperative information to the intraoperative situation. This problem is a very interesting and challenging research field.

Different methods have been proposed to solve the problem of the soft tissue deformation. In minimally invasive surgery, the methods are mainly based on the use of intraoperative imaging and tracking systems [8-10]. One solution to the deformation produced by the movement of the organ was described by Zhang et al. [11], who compensate organ motion using an electromagnetic tracking system. Despite all efforts, the simulation of deformations in some interventions such as liver surgery is a complicated task and actually some improvements are required [12]. Lamata et al. [12] propose an intermediate system for assisting minimally invasive liver resection. This approach shows the resection map preoperatively planned during surgery, allowing the surgeon to perform an alignment between the map of resection and the operating field. We think that the accurate intraoperative deformation can be achieved through the intervention of an assistant that will interact with the CAS system manually. However, it is necessary to have a iv) suitable profile to identify anatomical structures in different intraoperative images and operate a computer assisted surgery.

Another problem of the intraoperative navigation system is its management during surgery, as the surgeon can't interact directly with the system. Therefore, it is essential that the assistant operates with the system during surgery. It is also necessary that the surgeon's instructions are clearly interpretable and reproducible by the assistant in the navigation system [7], because otherwise it may hamper the intervention.

\subsection{Haptics \& Robotics}

Robotic Minimally Invasive Surgery (RMIS) is a high rising technique in the last years that enhances accuracy and safety of surgical procedures [14]. However, current robotic systems for surgery are not fully integrated with surgical planning and navigation systems. For this reason, pre- and intra-operative imaging guidance are one of the most important field of research needed to integrate the specific models of the patient with robotic systems or smart surgical tools. MRI mechanical manipulators [13] and visual servoing [14] techniques are shown as some of the most promising results, although all of these developments are focused on the visual sense as the main source of information for the surgeon. 
On the other hand, touch sense problems in MIS are well known [16] and some studies demonstrate that the lack of touch feedback in MIS can increase intra-operative injury [15]. Therefore, one important trend in RMIS could be considered as force feedback of robotic surgical systems that allows for surgeons to have similar touching sensations like in open surgery [17]. Furthermore, tactile feedback is another feature lacking in MIS that should be solved in future developments [18].

\section{THE CAS SYSTEM NURSE}

As mentioned previously Computer Assisted Surgery systems at a basic level allow for the display of live video images from the endoscopic camera and the display of preoperative images during the operation hence lessening the cognitive load of the surgeon. In the previous section we listed the following four problems in intra-operative imaging that we believe can be addressed with a new job profile, the computer assisted surgery nurse:

\section{i) Drastic morphological changes}

\section{ii) Superimposed over corresponding parts}

\section{iii) Partial views of organs}

\section{iv) Suitable profile}

In this section we present the CAS system nurse, and how this new job profile can address each of the four problems.

\section{Superimposed over corresponding parts}

Although existing 3D representations can have a supporting role during surgery, the inconsistencies from pre- and intra-operation could block the video information. To solve this, the models could be rendered in a separate viewport. The CAS nurse could then make real-time adjustments to the $3 \mathrm{D}$ reconstructed models such as scaling and rotation.

\section{Drastic morphological changes}

The CAS nurse could use sliders to control the transparency level of objects that no longer correspond to the intra-operative data. Ideally lines of a 3D grid could be superimposed over the rendered object (polygonal surface or volumetric render), and the CAS nurse could change the transparency level of each cube. Removed parts by the surgeon would be completely transparent. In the case of multiple enclosed surfaces of for example a tumour enclosed in the liver, the whole liver object could be selected by the CAS nurse and have its transparency increased in order for the surgeon to see the surface rendering of the tumour.

\section{Partial views of organs}

As mentioned earlier, tracking algorithms have significant difficulties to find correspondences with partial views of organs. We suggest that the human can beat these algorithms while they are not fully reliable yet, the CAS nurse can manually align the 3D models so as to have the same pose/orientation of the parts of organs that are indeed visible. The models could also be zoomed so as to focus the rendering in the working area.

\section{Suitable profile}

In order for the CAS nurse to be able to identify from video images organs with partial views, the CAS nurse would need to undergo several hours of training, attending real laparoscopic surgeries. 
The CAS nurse could respond to specific voice commands from the surgeon during the operation; such as changing the content of a particular viewport (e.g. Figure 4), access a preoperation file, zoom or un-zoom the 3D rendering, or rotate the 3D model.

These voice commands could be formalized, thus ensuring that the training for this job profile could be then used in different countries and hospitals.

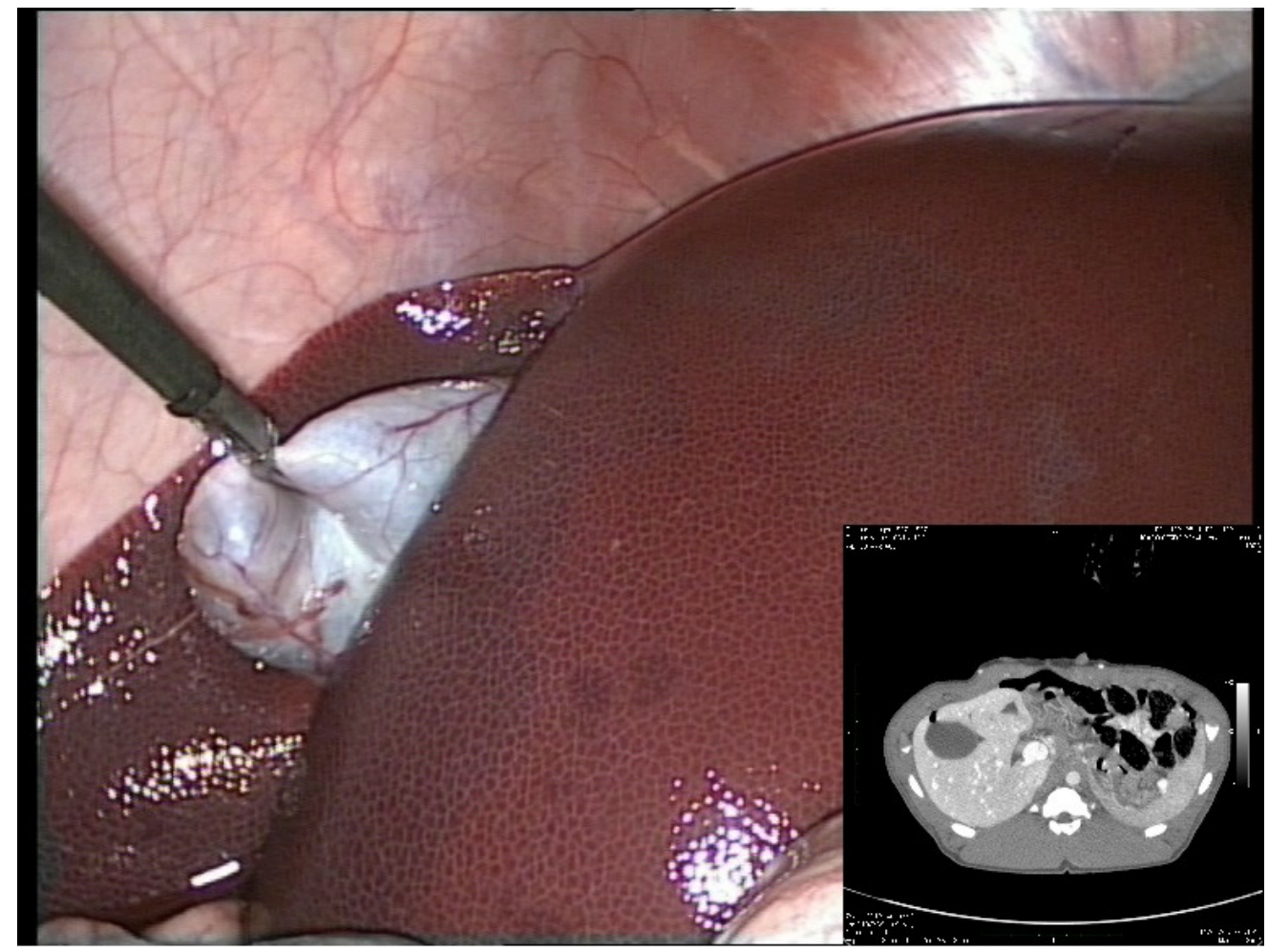

Figure 4. The CGI nurse overlays preoperative CT data over live intraoperative video images

\section{FUTURE WORK}

We are developing a new CAS system, where two intra-operating video feeds will be captured and displayed (ultrasound and endoscopic camera). The system will allow the CAS nurse to pause any of the feeds, it will have separate configurable viewports for the display of preoperation data, one of the viewports will allow the CAS nurse to rotate, and scale the 3D reconstructed organs to align them with the data from the video feed. Finally the system will enable the slice marking of veins, organs and tumours to create 3D reconstructions. We hope that these new capabilities of CAS nurse will help both the exploratory phase of the surgery (find the organ) and the actual surgery where resection maps and transparency control of removed parts should be possible.

\section{Conclusions}

We have reviewed some of the main problems of existing CAS systems for adapting preoperation data during the surgery. Four specific aspects were listed, and a new job profile that could address those aspects was presented. It is hoped that this new job profile could help in the operation room whilst existing techniques are further developed. 
International Journal on Bioinformatics \& Biosciences (IJBB) Vol.2, No.4, December 2012

\section{ACKNOWLEDGEMENTS}

The authors would like to thank the medical staff from Hospital Dr. José Maria Grande in Portalegre Portugal for their cooperation and Red de Investigación Transfronteriza de Extremadura Centro y Alentejo. This project is funded by Programa de Cooperación Transfronteriza España Portugal (POCTEP) and Fondo Europeo de Desarrollo Regional (FEDER) Reference code: 0401_RITECA_II_4_E.

\section{REFERENCES}

[1] da Luz Moreira, A. \& Kiran, R. P. \& Kirat, H. T. \& Remzi, F. H. \& Geisler, D. P. \& Church, J. M. \& Garofalo, T. \& Fazio, V. W., (2010) "Laparoscopic versus open colectomy for patients with American Society of Anesthesiology (ASA) classification 3 and 4: the minimally invasive approach is associated with significant quicker recovery and reduced costs", Surgical Endoscopy, Vol. 24, No. 6, pp1280-1286.

[2] Haigron, P. \& Dillenseger, J. L., (2010) "Image-guided therapy: evolution and breakthrough", IEEE Engineering in Medicine and Biology Magazine, Vol. 29, No. 1, pp100-104, doi:10.1109/MEMB.2009.935459.

[3] Boothroyd, A. \& etc, (2012) "Mesh cutting algorithm for an orthopaedic surgery simulator", blah

[4] De Novi, G., (2011) "Soft Tissue Modeling for Virtual Reality Surgery Simulator with Haptic Feedback", PhD Thesis, Università di Bologna.

[5] Peters, T. \& Cleary, K., (2008) 'Image-Guided Interventions", Technology and Applications, Springer, ISBN 978-0-387-73856-7.

[6] Doignon, C. \& Nageotte, F. \& Maurin, B. \& Krupa, A., (2008) "Pose estimation and Feature Tracking for Robot Assisted Surgery with Medical Imaging”, Unifying Perspectives in Computational and Robot Vision Lecture Notes in Electrical Engineering, Springer, Vol. 8, pp79-101.

[7] Baumhauer, M. \& Feuerstein, M. \& Meinzer, H.-P. \& Rassweiler, J., (2008) "Navigation in endoscopic soft tissue surgery: perspectives and limitations", Journal of endourology, Vol. 22, No.4, pp751-66, doi:10.1089/end.2007.9827.

[8] Ross, J.C. \& Tranquebar, R. \& Shanbhag, D., (2008) "Real-time liver motion compensation for MRGFUS”, Med Image Comput Assiste Interv, Vol 11, pp 806-813.

[9] Guckenberger, M. \& Richter, A. \& Boda-Heggemann, J. \& Lohr, F., (2012) "Motion compensation in radiotherapy", Crit Rev Biomed Eng, Vol. 40, No. 3, pp187-97.

[10] Arnold, P. \& Preiswerk, F. \& Fasel, B. \& Salomir, R. \& Scheffler, K. \& Cattin, PC., (2011) “3D organ motion prediction for MR-guided high intensity focused ultrasound”, Med Image Comput Comput Assist Interv, Vol. 14, pp 623-30.

[11] Zhang, H. \& Banovac, F. \& Lin, R. \& Glossop, N. \& Wood, B.J. \& Lindisch, D. \& Levy, E. Cleary K., (2006), "Electromagnetic tracking for abdominal interventions in computer aided surgery” Comput Aided Surg, Vol. 11, No. 3, pp127-36.

[12] Lamata, P. \& Lamata, F. \& Sojar, V. \& Makowski, P. \& Massoptier, L. \& Casciaro, S. \& Ali, W. \& Stüdeli, T. \& Declerck \& Elle, O. J. \& Edwin, B. (2010) "Use of the Resection Map system as guidance during hepatectomy”, Springer Surgical Endoscopy, Vol. 24, No. 9.

[13] Tsekos, N. V. \& Khanicheh, A., Christoforou, E. \& Mavroidis C., (2007) "Magnetic resonancecompatible robotic and mechatronics systems for image-guided interventions and rehabilitation: a review study", Annual review of biomedical engineering, Vol. 9, No. 5, pp351-87, doi:10.1146/annurev.bioeng.9.121806.160642.

[14] Ed. Bozovic, V., (2008) "Medical Robotics", I-Tech Education and Publishing, ISBN 978-3902613-18-9. 
International Journal on Bioinformatics \& Biosciences (IJBB) Vol.2, No.4, December 2012

[15] Xin, H. \& Zelek, J. S. \& Carnahan, H., (2006) "Laparoscopic surgery, perceptual limitations and force: A review", In First Canadian Student Conference on Biomedical Computing, Kingston, Ontario, Canada, 2006. No. 144.

[16] Westebring-van der Putten, E. P. \& Goossens, R. H. \& Jakimowicz, J. J. \& Dankelman, J., (2008) "Haptics in minimally invasive surgery--a review", Minim Invasive Ther Allied Technol. Vol. 17, No. 1, pp3-16.

[17] Okamura, A. M. \& Verner, L. N. \& Reiley, C. E. \& Mahvash, M., (2007) "Haptics for RobotAssisted Minimally Invasive Surgery", In 13th International Symposium of Robotics Research (ISRR 2007), Hiroshima, Japan, 2007.

[18] Nguan, C. \& Girvan, A. \& Luke, P. P., (2008) "Robotic surgery versus laparoscopy: a comparison between two robotic systems and laparoscopy", J Robotic Surg, 1:263-268, doi:10.1007/s11701-007-0050-x.

\section{Authors}

João Fradinho Oliveira holds a $\mathrm{PhD}$ in Computer Science (Geometry reduction) from University College London. He finished his post-doc in outof-core visualization techniques at INESC-ID (Computer Systems Engineering Institute) in 2011. He is currently adjunct Professor at Instituto Politécnico de Portalegre, Escola Superior de Tecnologia e Gestão, where he teaches 3D programming. His main interests are point cloud processing and 3D visualization systems. He has published 18 papers.

Hugo Chichorro e Silva Capote graduated in Medicine, in 2001, in the Medical School of the Coimbra University, Portugal. In 2009, after 6 years of formation in the Hospital of Portalegre he obtained the degree of Assistant in the specialty of General Surgery. Since then he works as a Surgeon in Portalegre, being one of the staff that is responsible for the Coloproctology Unit of the Department of Surgery. He works routinely with laparoscopy, performing several different techniques, after having trained in Strasbourg at IRCAD and Cáceres at CCMI. He has published papers on different subjects, such as colon and small bowel pathology and some clinical cases.

Jose Blas Pagador is the Head Manager of the Bioengineering and Health Technologies Unit within Jesús Usón Minimally Invasive Surgery Centre. He is a Master Engineer in Computer Science from the University of Extremadura, specialized on Minimally Invasive Surgery Devices and Technologies. One of his main research interests is focused on surgical training and assessment. He has participated in 21 research projects and he has published 16 papers and more than 50 communications. He has organized 2 International Scientific Meetings

Jose Luis Moyano Cuevas. Obtained his Informatic Engineer degree in 2007 at the University of Extremadura where he got a research fellowship on mobile technologies. In 2012, he obtained the Master Engineer in Computer Science at University of Extremadura. Since 2008, he works as a researcher at the Bioengineering and Health Technologies Unit at Jesús Usón Minimally Invasive Surgery Centre. His main research lines are medical image and the computer assisted surgery. He has participated in five research projects and he
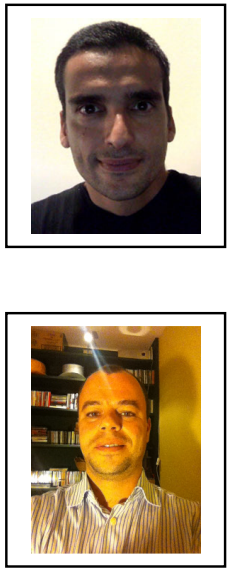
is co-author of the 7 publications in journals.
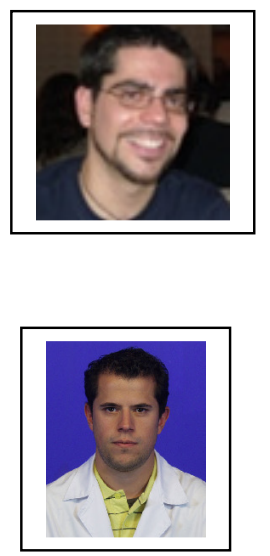
International Journal on Bioinformatics \& Biosciences (IJBB) Vol.2, No.4, December 2012

Francisco Miguel Sánchez Margallo is Scientific Director of the Jesús Usón Minimally Invasive Surgery Centre and Doctor of Veterinary Science at the University of Extremadura. Instructor in more than 400 training activities in minimally invasive surgery. Professor and organizer of more than 375 national and international courses for practical training of surgeons and health professionals. Author of 86 scientific articles published in magazines with Impact Factor and author and co-author of more than 79 articles of diffusion related to Minimally Invasive Surgery and Surgery. Author of 10 books and co-author of 45 book chapters. Author of 100 scientific posters, over 300 invited lectures and free communications presented at national and international congresses. He has participated in 67 research projects funded by public entities and companies. He is also member of several scientific and organization committees in different symposium and conferences, as well as reviewer of scientific journals.

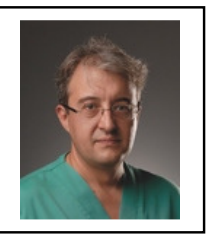

